\title{
ARTICLE OPEN Competing magnetostructural phases in a semiclassical system
}

\author{
Kenneth R. O'Neal ${ }^{1}$, Jun Hee Lee ${ }^{2}$, Maeng-Suk Kim ${ }^{2}$, Jamie L. Manson ${ }^{3}$, Zhenxian Liu ${ }^{4}$, Randy S. Fishman ${ }^{5}$ and Janice L. Musfeldt ${ }^{1,6}$
}

The interplay between charge, structure, and magnetism gives rise to rich phase diagrams in complex materials with exotic properties emerging when phases compete. Molecule-based materials are particularly advantageous in this regard due to their low energy scales, flexible lattices, and chemical tunability. Here, we bring together high pressure Raman scattering, modeling, and first principles calculations to reveal the pressure-temperature-magnetic field phase diagram of $\mathrm{Mn}\left[\mathrm{N}(\mathrm{CN})_{2}\right]_{2}$. We uncover how hidden soft modes involving octahedral rotations drive two pressure-induced transitions triggering the low $\rightarrow$ high magnetic anisotropy crossover and a unique reorientation of exchange planes. These magnetostructural transitions and their mechanisms highlight the importance of spin-lattice interactions in establishing phases with novel magnetic properties in $\mathrm{Mn}$ (II)-containing systems.

npj Quantum Materials (2017)2:65; doi:10.1038/s41535-017-0065-0

\section{INTRODUCTION}

The interplay between charge, structure, and magnetism has a profound effect on the functionality of complex materials. ${ }^{1-4}$ These interactions are particularly strong in oxides, giving rise to elaborate $P-T-B-h v$ phase diagrams, often with exotic and/or coexisting states that derive from delicately balanced coupling. ${ }^{5-8}$ External perturbations such as pressure, magnetic field, or light, can change important energy scales, driving these flexible materials into new areas of phase space with very different properties. This is interesting because exotic properties tend to emerge when phases compete. While of foundational importance, phase transitions driven by external stimuli can be difficult to realize at experimentally attainable conditions in specific physical systems. ${ }^{9-12}$ This is because material energy scales tend to be high relative to the size of an external tuning parameter like pressure or magnetic field. As a result, investigations often focus on metal-insulator transitions in organic or oxide materials ${ }^{13-16}$ and superconducting systems. ${ }^{17-19}$ Molecule-based materials, with their flexible lattices, overall low energy scales, and chemical tunability, ${ }^{20-23}$ provide an alternate approach to the exploration of pressure- and magnetic field-induced transitions. At the same time, they provide opportunities to isolate spin-lattice interactions and test the chemical aspects of these processes-in this case, the ability of high spin $S=5 / 2 \mathrm{Mn}$ (II) centers to support novel states of matter.

The $M\left[\mathrm{~N}(\mathrm{CN})_{2}\right]_{2}(M=\mathrm{Ni}, \mathrm{Co}, \mathrm{Mn}, \mathrm{Fe})$ family of materials provides a superb platform for exploring phases that arise under external stimuli. ${ }^{24-33} \mathrm{Mn}\left[\mathrm{N}(\mathrm{CN})_{2}\right]_{2}$ is a particularly important member of this series. ${ }^{34-38}$ In the Pnnm structure, the $\mathrm{Mn}$ (II) centers are connected by soft $\mathrm{N} \equiv \mathrm{C}-\mathrm{N}$ ligands that act as magnetic superexchange pathways. ${ }^{27}$ Below $T_{\mathrm{N}}=15.9 \mathrm{~K}$, the system is a weakly canted antiferromagnet. ${ }^{34}$ Magnetic field drives a spin-flop transition near $0.5 \mathrm{~T}$ and a transition to the fully saturated state at $30 \mathrm{~T}$ that is supported by dicyanamide distortions. ${ }^{30} \mathrm{~A}$ series of room temperature, pressure-induced structural transitions was recently discovered, ${ }^{38}$ although a full pressure-temperature $(P-T)$ phase diagram could not be developed from the data. This prevented an analysis of the thermodynamics and various phase relationships such as the precise connection between the lattice distortions and the low-temperature enhancement of magnetic anisotropy above $0.7 \mathrm{GPa}^{37}$ Reaching beyond a simple room temperature understanding of various structural phases is also crucial to exploring mechanistic scenarios. The relationship between the magnetostructural phases and $\mathrm{MnN}_{6}$ octahedral rotations also merits further investigation because unstable rotations and distortions are significant routes to ferroelectricity. ${ }^{39}$

In this work, we combine high pressure Raman spectroscopy with complementary modeling, lattice dynamics calculations, and prior magnetization ${ }^{30,37}$ to unveil the extremely rich pressure-temperature-magnetic field $(P-T-B)$ phase diagram of $\mathrm{Mn}\left[\mathrm{N}(\mathrm{CN})_{2}\right]_{2}$. We use this phase diagram-which reaches extremes in pressure and magnetic field that are rarely brought together-to untangle the connection between the different competing states. Tracking the series of pressure-induced phase transitions to low temperature reveals a delicate balance between magnetostructural phases, and our analysis of the octahedral rotations reveals the precise mechanism by which the different magnetic states are triggered. Enhanced exchange anisotropy above $0.7 \mathrm{GPa}^{37}$ for instance, is driven by softening of the octahedral rotation around $b$. Compression also stabilizes a previously unexplored soft mode-driven magnetic phase above 2.8 GPa that involves octahedral rotation around $a$ and is predicted to occur with a unique $90^{\circ}$ reorientation of the exchange planes. In addition to opening an avenue for closer examination of materials containing high spin $S=5 / 2 \mathrm{Mn}(\mathrm{II}),{ }^{40-42}$ these findings underscore the importance of cooperative structural distortions in the stabilization of novel magnetic phases and help to provide a strategy for the control of functional materials.

\footnotetext{
'Department of Chemistry, University of Tennessee, Knoxville, TN 37996, USA; ${ }^{2}$ School of Energy and Chemical Engineering, Ulsan National Institute of Science and Technology, Ulsan 44919, Korea; ${ }^{3}$ Department of Chemistry and Biochemistry, Eastern Washington University, Cheney, WA 99004, USA; ${ }^{4}$ Department of Civil and Environmental Engineering, The George Washington University, Washington DC 20052, USA; ${ }^{5}$ Materials Science and Technology Division, Oak Ridge National Lab, Oak Ridge, TN 37830, USA and ${ }^{6}$ Department of Physics, University of Tennessee, Knoxville, TN 37996, USA

Correspondence: Jun Hee Lee (junhee@unist.ac.kr) or Janice L. Musfeldt (musfeldt@utk.edu)
}

Received: 17 March 2017 Revised: 19 September 2017 Accepted: 12 October 2017

Published online: 22 November 2017 

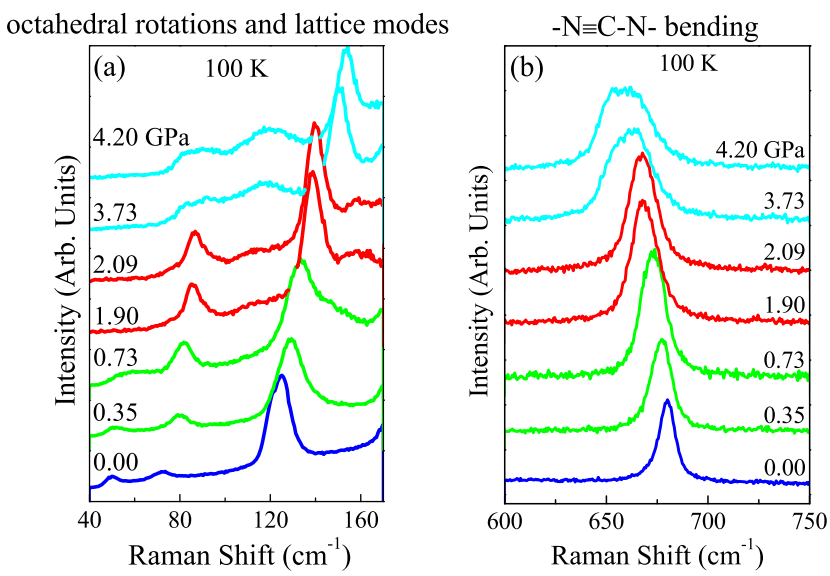

$\mathrm{C} \equiv \mathrm{N}$ stretch and overtone mode
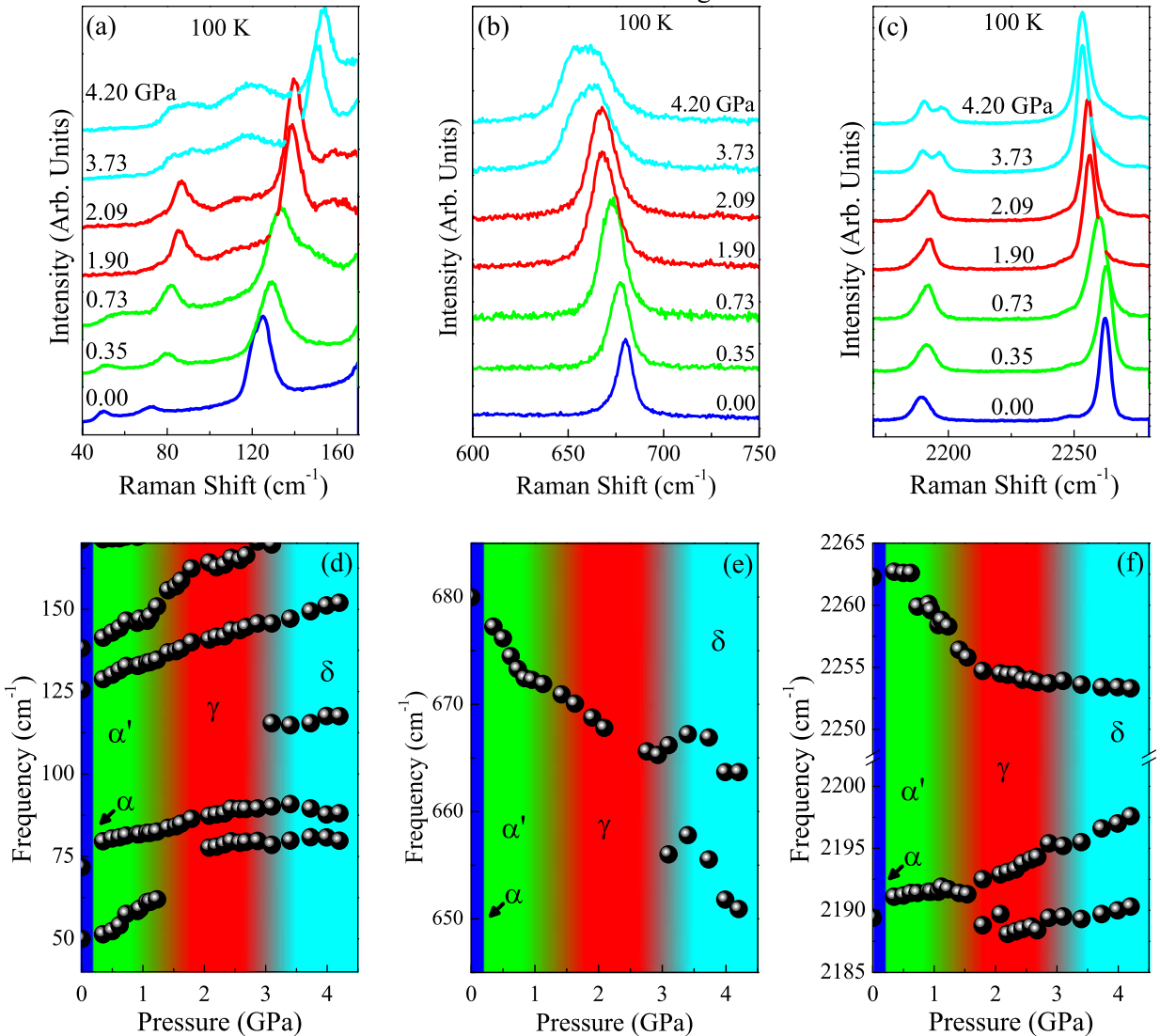

Fig. 1 a-c Close-up views of the $100 \mathrm{~K}$ Raman spectra of $\mathrm{Mn}\left[\mathrm{N}\left(\mathrm{CN}_{2}\right)\right]_{2}$ as a function of pressure. d-f Frequency vs. pressure for the modes displayed in a-c from which we determine the critical pressures. Line and background colors correlate with well-defined phases. Here, blue, green, red, and cyan represent the ambient pressure orthorhombic (Pnnm) $a$ phase and the high pressure $\alpha^{\prime}, \gamma$, and $\delta$ phases, respectively. The blending near $P_{\mathrm{C} 2}$ and $P_{\mathrm{C} 3}$ indicates the range over which the structural distortions take place

\section{RESULTS}

Revealing structural phase transition mechanisms

Figure 1 displays close-up views of the Raman response of $\mathrm{Mn}[\mathrm{N}$ $\left.(\mathrm{CN})_{2}\right]_{2}$ as a function of pressure at $100 \mathrm{~K}$. The majority of features display traditional compression-induced hardening, ${ }^{38}$ so we focus on the behavior of the octahedral rotations, the lowest frequency lattice modes, the $\mathrm{C} \equiv \mathrm{N}-\mathrm{C}$ ligand bend, and the $\mathrm{C} \equiv \mathrm{N}$ stretch as most significant. Peak position vs. pressure plots along with a frequency shift and splitting pattern analysis reveal a series of pressure-induced structural phase transitions at $0.2,1.3$, and 3.0 GPa. In order to simplify our discussion of the structural distortions, we designate the phases as $a, a^{\prime}, \gamma$, and $\delta$ from lowest to highest pressure. The sharp $a \rightarrow a^{\prime}$ transition at $P_{\mathrm{C} 1}=0.2 \mathrm{GPa}$ takes place within the orthorhombic $P n n m$ space group. It involves only local distortions in the $\mathrm{MnN}_{6}$ environment as indicated by a hardening of the $50 \mathrm{~cm}^{-1}$ mode associated with octahedral rotation around $c$ and a change in slope of the $80 \mathrm{~cm}^{-1}$ equatorial out-of-phase $\mathrm{N}-\mathrm{Mn}-\mathrm{N}$ bending mode. The more gradual $a^{\prime} \rightarrow \gamma$ crossover at $P_{\mathrm{C} 2}=1.3 \mathrm{GPa}$ is signaled by the disappearance of the $50 \mathrm{~cm}^{-1}$ octahedral rotation around $c$ and splitting of the $2190 \mathrm{~cm}^{-1} \mathrm{C} \equiv \mathrm{N}$ stretch. Finally, the $\gamma \rightarrow \delta$ crossover near $P_{\mathrm{C} 3}=3.0 \mathrm{GPa}$ is identified by splitting of the mode at 120 $\mathrm{cm}^{-1}$, a significant width enhancement of the $\mathrm{C} \equiv \mathrm{N}-\mathrm{C}$ bending mode near $680 \mathrm{~cm}^{-1}$ that we attribute to weak splitting, and rigidity in the $2260 \mathrm{~cm}^{-1}$ overtone. As discussed below, $P_{\mathrm{C} 2}$ and $P_{C 3}$ have important effects on the crystal symmetry and magnetic properties. $^{38}$
We extended these variable pressure Raman scattering measurements and the analysis shown in Fig. 1 to include several temperatures between 100 and $300 \mathrm{~K}$. In each case, we identify a series of three critical pressures. As before, the $a \rightarrow a$ transition is sharp whereas the $a^{\prime} \rightarrow \gamma$ and $\gamma \rightarrow \delta$ crossovers take place continuously over much broader pressure ranges. Figure 2 displays the pressure-temperature $(P-T)$ phase diagram of $\mathrm{Mn}[\mathrm{N}$ $\left.(\mathrm{CN})_{2}\right]_{2}$. We immediately notice that the phase boundaries corresponding to these structural distortions have positive $\partial P /$ $\partial T$ s. Since $(\partial P / \partial T)_{V}=-\left(\partial S / \partial V_{T}\right.$, the slopes indicate that entropy is increasing-becoming more disordered through each successive structural transition. These phase boundaries drive toward an interesting set of magnetic states at low temperature.

In order to unveil the mechanism behind this series of structural phase transitions, we performed first-principles calculations of $\mathrm{Mn}$ $\left[\mathrm{N}(\mathrm{CN})_{2}\right]_{2}$ under isotropic compression and compared our predictions with the experimental results (Fig. $3 \mathrm{a}, \mathrm{b}$ ). Our calculations reveal three octahedral rotational modes (Fig. $3 \mathrm{c}-\mathrm{e}$ ), two of which strikingly become unstable under pressure. These soft modes drive permanent lattice distortions and are responsible for the structural phase transitions at $P_{\mathrm{C} 2}$ and $P_{\mathrm{C} 3}$ in $\mathrm{Mn}\left[\mathrm{N}(\mathrm{CN})_{2}\right]_{2}$. By contrast, the transition at $P_{C_{1} 1}$ is a local distortion and therefore is not associated with a soft mode. Thus, while $P_{\mathrm{C} 2}$ and $P_{\mathrm{C} 3}$ are amenable to $\mathrm{x}$-ray investigation, $P_{\mathrm{C} 1}$ can not be resolved by an average structure technique. The soft modes predicted to appear at 49 and $53 \mathrm{~cm}^{-1}$ are unfortunately not observed in our experiments because (i) the features overlap and (ii) the calculated matrix elements are very small ( 0.018 and 4.43 compared to $\approx 44$ 


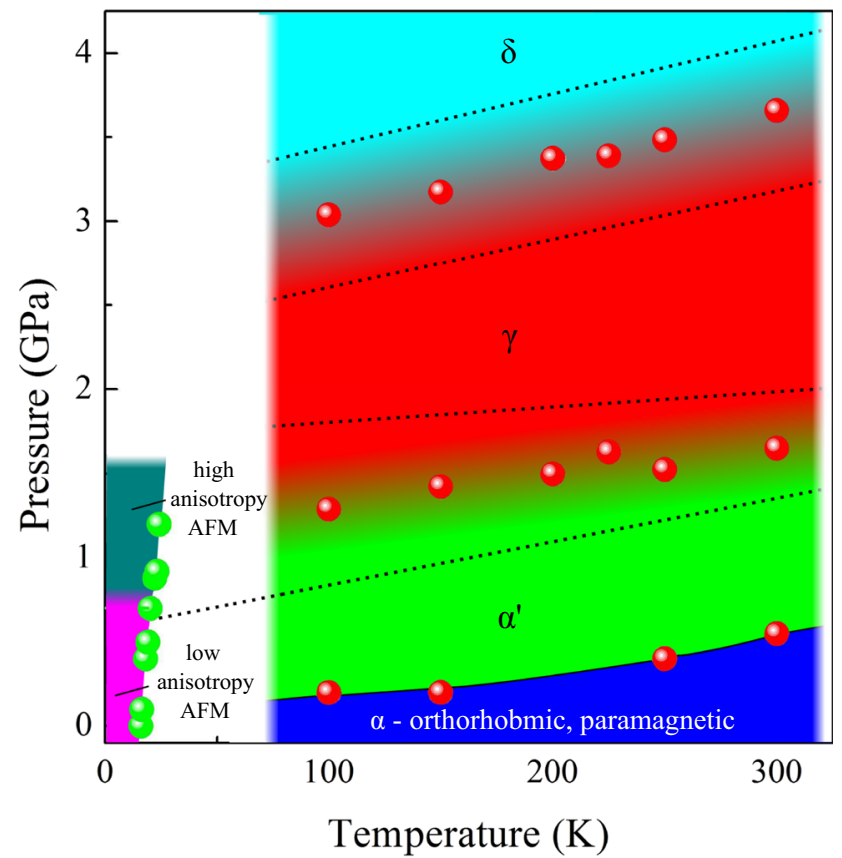

Fig. 2 Pressure-temperature phase diagram of $\mathrm{Mn}\left[\mathrm{N}(\mathrm{CN})_{2}\right]_{2}$ obtained by combining the critical pressures determined by Raman scattering (red points) with magnetic properties data (green points) from Quintero et al. $^{37}$ The blue, green, red, and teal regions correspond to the $a, a^{\prime}, \gamma$, and $\delta$ phases, respectively. The breadth of the phase boundaries near $P_{\mathrm{C} 2}$ and $P_{\mathrm{C} 3}$ is indicated by blending between the dotted lines for the $120 \mathrm{~cm}^{-1}$ lattice mode). This highlights the role of predictive theory in unraveling precisely how and why a phase transition takes place.

Overall, the predicted critical pressures are in excellent agreement with the sequence of structure phase transitions displayed in the $P-T$ phase diagram (Fig. 2). The octahedral rotation around $b$ ( $R_{b}$ in Fig. $3 c$ ) is predicted to become imaginary at $2.2 \mathrm{GPa}$, near the observed $a^{\prime} \rightarrow \gamma$ crossover at $P_{\mathrm{C} 2}=1.3 \mathrm{GPa}$. The $\mathrm{MnN}_{6}$ rotation about the $a$ axis $\left(\mathrm{R}_{a}\right.$ in Fig. $\left.3 \mathrm{~d}\right)$ is predicted to go soft at $3.1 \mathrm{GPa}$, very close to the $\gamma \rightarrow \delta$ crossover at $P_{\mathrm{C} 3}=3.0$ GPa. Finally, we point out that the ambient structure is already displaced through the octahedral rotation around $c\left(\mathrm{R}_{c}\right.$ in Fig. 3e), and therefore the $\mathrm{MnN}_{6}$ rotational mode observed at $50 \mathrm{~cm}^{-1}$ does not go soft (Fig. 3a, b). Trends in the lattice and localized modes are reproduced as well, ${ }^{38}$ although small discrepancies arise from carrying out our calculations under constant symmetry.

\section{Structural phase transitions trigger new magnetic states}

This series of pressure-driven structural distortions has important implications for low temperature magnetism. The connection is readily apparent from the $P-T$ phase diagram, where we see that $\mathrm{Mn}\left[\mathrm{N}(\mathrm{CN})_{2}\right]_{2}$ sports at least two long-range ordered magnetic states that differ in their anisotropy. ${ }^{37}$ Extrapolating the pressureinduced structural transitions (the edges of which are indicated by dotted lines in Fig. 2) to base temperature reveals that the low pressure edge of the $a^{\prime} \rightarrow \gamma$ transition directly encounters the magnetic phase boundary. This coincidence suggests that it triggers the anisotropy crossover. Despite the temperature limitations imposed by our in-situ pressure experiments, this sort of direct connection from simple inspection of the phase diagram is not often apparent. Our calculations link the $a^{\prime} \rightarrow y$ structural phase transition and the magnetic anisotropy crossover as well.
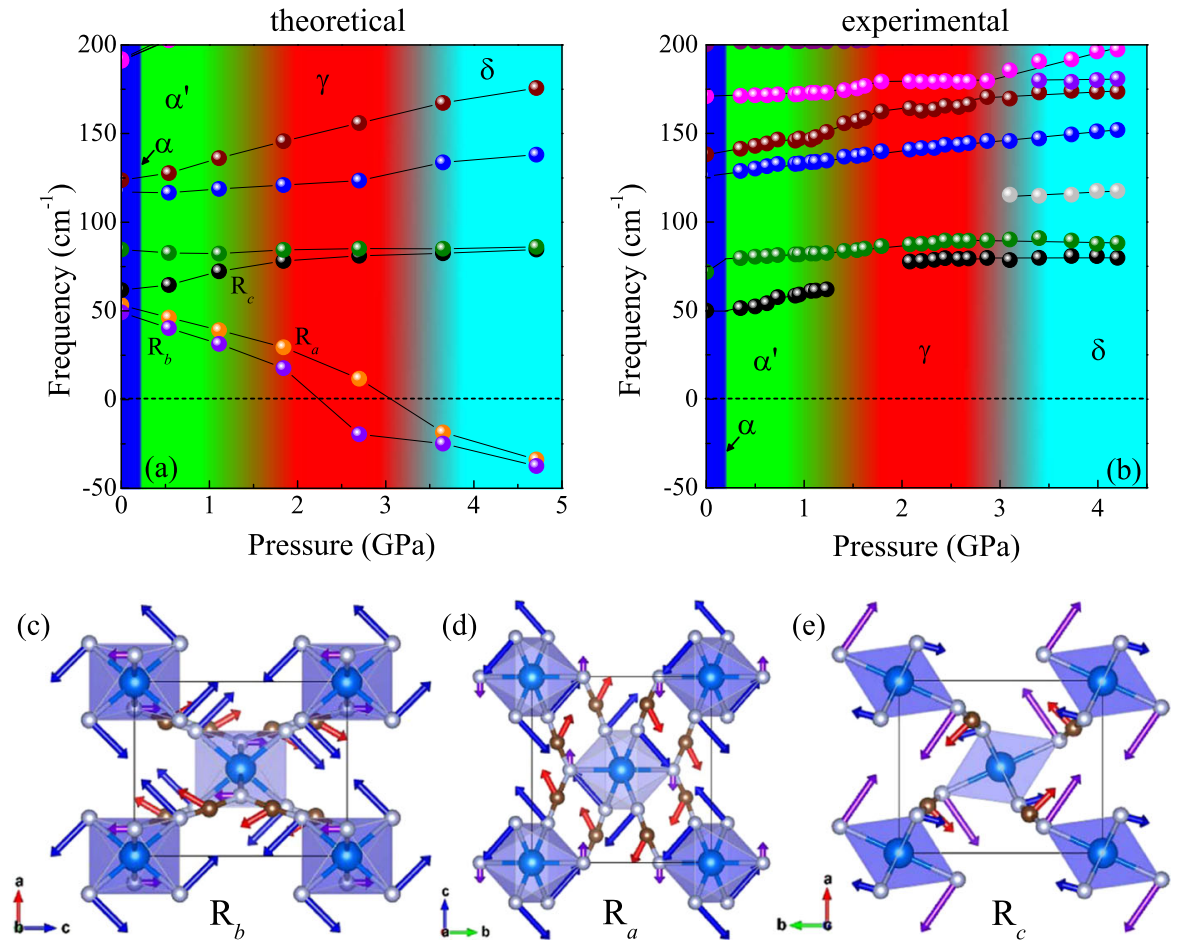

Fig. 3 (a) Calculated and (b) experimental frequency vs. pressure trends for the low frequency Raman-active modes of $\mathrm{Mn}\left[\mathrm{N}(\mathrm{CN})_{2}\right]_{2}$. Colored regions correspond to various phases as labeled, with blended areas indicating gradual crossovers. c-e Displacement patterns for the octahedral rotation modes around $b, a$, and $c$ 

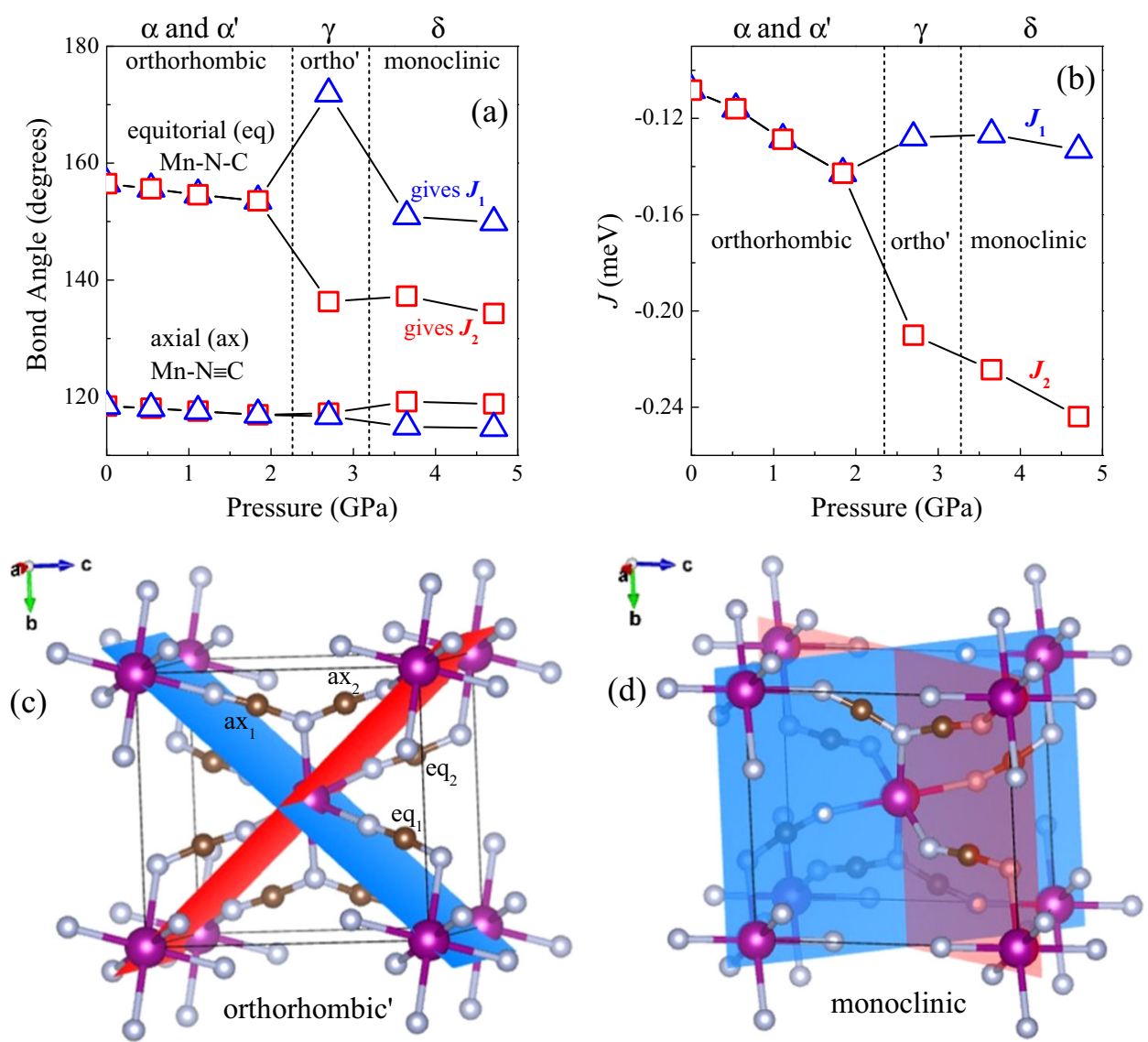

Fig. 4 Calculated (a) $\mathrm{Mn}-\mathrm{N} \equiv \mathrm{C}$ and $\mathrm{Mn}-\mathrm{N}-\mathrm{C}$ angles and (b) $J$ vs. pressure. $J_{1}\left(J_{2}\right)$ is the exchange interaction of spins located in the blue (red) plane of (c) the orthorhombic' $\gamma$ and (d) monoclinic $\delta$ phases. The exchange interactions in both the $\gamma$ and $\delta$ phases support G-type antiferromagnetism on a bipartite lattice. The blue and red magnetic planes (associated with $J_{1}$ and $J_{2}$ ) rotate by $90^{\circ}$ in a cooperative manner across the structural transition

Figure 4 displays two of the important angles in the $\mathrm{Mn}-\mathrm{N} \equiv \mathrm{C}-\mathrm{N}-\mathrm{Mn}$ superexchange pathway and the corresponding exchange interaction between metal centers $(J)$ as a function of pressure. These values are computed in two different magnetic planes. Strikingly, we predict the development of two unique $\mathrm{Mn}-\mathrm{N} \equiv \mathrm{C}-\mathrm{N}-\mathrm{Mn}$ bridges under pressure (in agreement with our spectral findings), an unprecedented change in the $\mathrm{Mn}-\mathrm{N}-\mathrm{C}$ bond angle (by more than $\pm 20^{\circ}$ ), and a consequent splitting of $J$ in the $\gamma$ phase. These two different exchange interactions arise due to the octahedral rotations. Although we predict that the system remains orthorhombic, the development of two unique $J$ values is consistent with a change in exchange anisotropy. We therefore conclude that structural distortions across the $a^{\prime} \rightarrow \gamma$ transition drive the anisotropy enhancement ${ }^{37}$ in the $\gamma$ phase.

The temperature dependence of the $\gamma \rightarrow \delta$ transition provides compelling evidence for an unexplored low temperature magnetic phase near $3 \mathrm{GPa}$. Our calculations predict that the $53 \mathrm{~cm}^{-1}$ soft mode $\left[R_{a}\right]$ drives the system to a monoclinic space group, consistent with the appearance of new spectral features. As seen in Fig. $4 \mathrm{a}$, the $120^{\circ} \mathrm{Mn}-\mathrm{N} \equiv \mathrm{C}$ angle splits into two, giving a total of four unique superexchange angles in the $\delta$ phase. This is also in line with splitting of the $\mathrm{N} \equiv \mathrm{C}-\mathrm{N}$ ligand bending mode around 680 $\mathrm{cm}^{-1}$ (Fig. $1 \mathrm{~b}, \mathrm{e}$ ). The $\delta$ phase thus displays two unique $J$ values (Fig. 4b) that become more distinct under compression. Another intruiging aspect of the $\gamma \rightarrow \delta$ transition in $\mathrm{Mn}\left[\mathrm{N}(\mathrm{CN})_{2}\right]_{2}$ is that it takes place with a $90^{\circ}$ reorientation of the exchange planes (Fig. 4c, d). According to our calculations, this unusual exchange plane reorientation is a consequence of the $90^{\circ}$ separation of the rotation axes of the $\mathrm{R}_{b}$ and $\mathrm{R}_{a}$ soft modes that define the structural phases. High pressure magnetization or inelastic neutron scattering may be able to detect these exotic changes in the microscopic spin rearrangement.

\section{DISCUSSION}

Pressure and temperature are not the only tuning parameters that support new states of matter in $\mathrm{Mn}\left[\mathrm{N}(\mathrm{CN})_{2}\right]_{2}$. Applied magnetic field drives a spin-flop transition ${ }^{35}$ and ultimately a transition to the fully polarized state that is stabilized by lattice distortions. ${ }^{36}$ Here, field suppresses the quantum fluctuations associated with the canted antiferromagnetic state to saturate the magnetization above $30 \mathrm{~T}$. Bringing these results together with our current effort yields an extraordinarily rich $P-T-B$ phase diagram (Fig. 5) with a surprising number of competing magnetostructural phases. This complexity arises from the delicate balance of interactions in $\mathrm{Mn}$ $\left[\mathrm{N}(\mathrm{CN})_{2}\right]_{2}$, making it possible for small external perturbations to modify the important energy scales and mixing processes and driving the system into new phases with unique properties. The low $\rightarrow$ high anisotropy transition with pressure and field (taking the system from Heisenberg to Ising-like) is an especially nice illustration of pressure/field complementarity. The ability to explore materials in these extreme environments is obviously very unusual. What makes this particular $P-T-B$ phase diagram especially fascinating is the prospect that these phases may host emergent properties at experimentally-realizable conditions. An important aspect of the phase diagram in Fig. 5 is that the majority of transitions depend upon external stimuli rather than thermal fluctuations. We argue here that the mechanism for the 


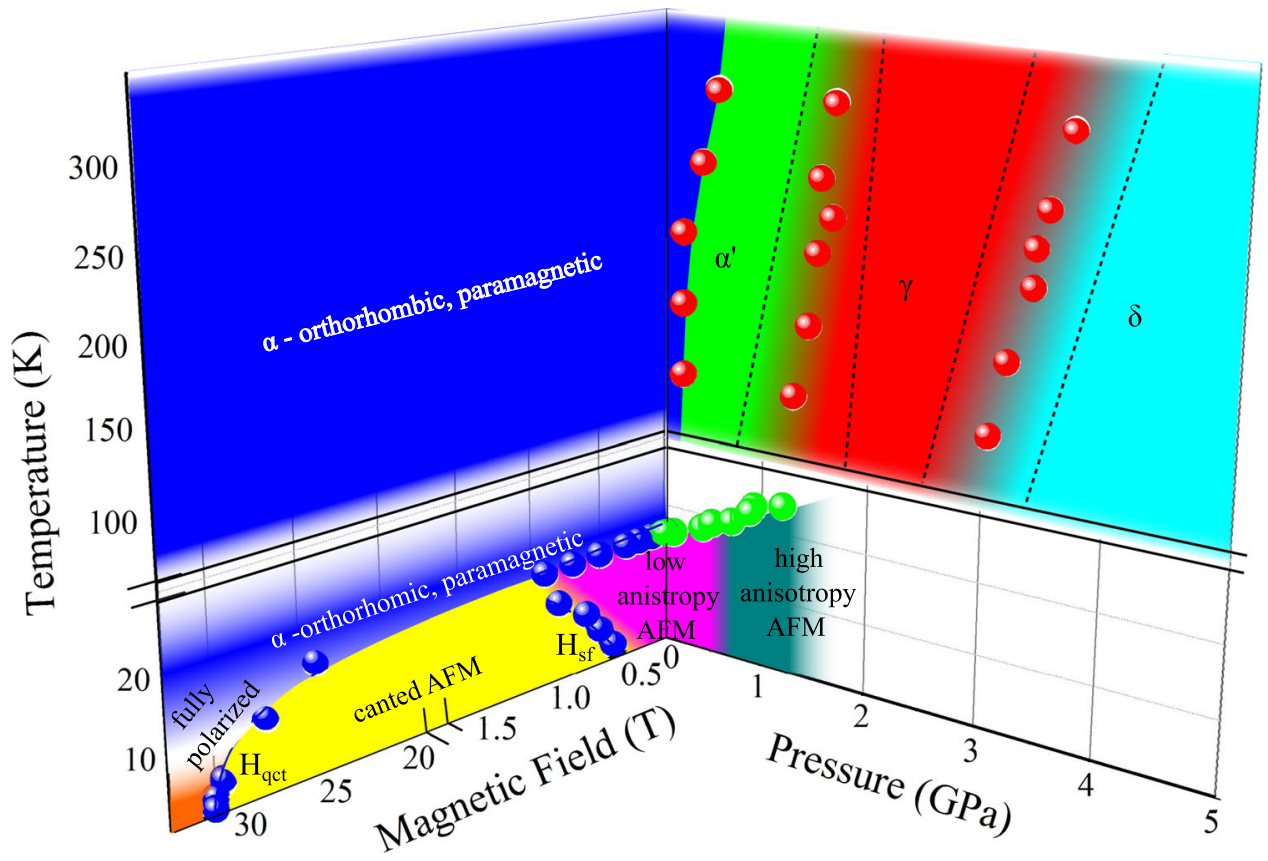

Fig. 5 Pressure-temperature-magnetic field phase diagram of $\operatorname{Mn}\left[\mathrm{N}(\mathrm{CN})_{2}\right]_{2}$ created by combining our findings with prior magnetic and

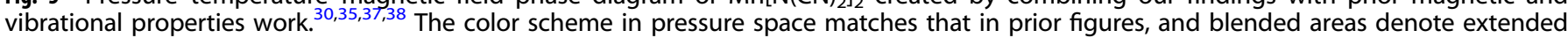
transition regimes. The structural and magnetic phases are labeled

pressure-induced transitions in $\mathrm{Mn}\left[\mathrm{N}(\mathrm{CN})_{2}\right]_{2}$ at $T=0 \mathrm{~K}$ depends on quantum fluctuations which are enhanced by softening of the phonon modes. Of course, high spin $\mathrm{Mn}$ (II) is typically described as a semi-classical or solely classical ion because spin is large $(S=5 / 2)$, single ion anisotropy is low, and quantum fluctuations (which go as $1 / S$ ) tend to be small-especially compared to $\mathrm{Mn}$ (III) and $\mathrm{Mn}(\mathrm{IV}){ }^{43}$ That said, dicyanimide is a high anisotropy ligand and is intimately involved in establishing the different Neél states under pressure. Moreover, our calculations predict that Dzyaloshinskii-Moriya interactions grow with decreasing bond angle (Fig. 4a). The magnetostructural phase diagram and mechanisms revealed here thus suggest that a number of the states and phase transitions in $\mathrm{Mn}\left[\mathrm{N}(\mathrm{CN})_{2}\right]_{2}$ may have unexpected complexity-especially above $P_{\mathrm{C} 3}$, where we predict a unique reorientation of exchange planes.

We emphasize that the magnetostructural coupling mechanisms discussed here are intrinsic to molecular materials and, in addition to being important features of $\mathrm{Mn}\left[\mathrm{N}(\mathrm{CN})_{2}\right]_{2}$, likely underlie the properties of hybrid transition metal-organic materials-many of which also contain $\mathrm{Mn}(\mathrm{II})$. Multiferroic $\left[\left(\mathrm{CH}_{3}\right)_{2} \mathrm{NH}_{2}\right] \mathrm{Mn}(\mathrm{HCOO})_{3}$ is a superb example. ${ }^{44-47} \mathrm{Mn}(\mathrm{TCNE})$ $\left[\mathrm{C}_{4}(\mathrm{CN})_{8}\right]_{1 / 2}$ and $\mathrm{Mn}_{1-x} \mathrm{Co}_{x} \mathrm{WO}_{4}$, which display a pressure-induced antiferromagnetic $\rightarrow$ ferrimagnetic transition and an eccentric spin cycloid respectively, also merit investigation in this regard. ${ }^{40,42}$ The competing phases in these and other materials $^{41,48-51}$ suggest that $\mathrm{Mn}(\mathrm{II})$-containing systems may provide an interesting new platform for exploring novel states of matter, some of which may even host quantum properties.

\section{METHODS}

Polycrystalline $\mathrm{Mn}\left[\mathrm{N}(\mathrm{CN})_{2}\right]_{2}$ was synthesized as described previously ${ }^{24}$ and loaded into a diamond anvil cell with an annealed ruby ball to determine pressure. ${ }^{52}$ Tests of loading conditions (either neat or with vacuum grease, liquid argon, or $\mathrm{KBr}$ as pressure media) demonstrate consistent results due to the soft nature of the material. Raman measurements were performed with a $532 \mathrm{~nm}$ diode pumped solid state laser, with $<1 \mathrm{~mW}$ power to prevent sample damage. Raman spectra were collected with a resolution of $0.5 \mathrm{~cm}^{-1}$, integrated between 60 and $120 \mathrm{~s}$, and averaged three times. An open-flow helium cryostat provided temperature control. In order to apply pressure in situ at low temperature, the diamond anvil cell was placed inside of a cryostat, the thermal contact of which limited our temperature range to $100 \mathrm{~K}$. Pressure was raised to $5 \mathrm{GPa}$ and fully released after each compression so that the material could relax. Standard peak fitting procedures were employed as appropriate.

First-principles calculations were performed based on density-functional theory within the generalized gradient approximation GGA $+U$ scheme ${ }^{53}$ with the Perdew-Becke-Erzenhof parametrization ${ }^{54}$ as implemented in the Vienna ab initio simulation package. ${ }^{55,56}$ We employed the Dudarev ${ }^{57}$ implementation with on-site Coulomb interaction $U=5 \mathrm{eV}$ to treat the localized $d$ electron states of $\mathrm{Mn}$. Within $\mathrm{GGA}+U$ this choice gives excellent agreement between the calculated $\left(4.66 \mu_{\mathrm{B}}\right)$ and experimental magnetic moments $\left(4.65 \mu_{\mathrm{B}}\right)$. $^{34}$ The projector augmented wave potentials $s^{58,59}$ explicitly include 13 valence electrons for manganese $\left(3 p^{6} 3 d^{5} 4 s^{2}\right)$, 5 for nitrogen $\left(2 s^{2} 2 p^{3}\right)$, and 4 for carbon $\left(2 s^{2} 2 p^{2}\right)$. Structural optimizations were performed for a 22 -atom unit-cell with a $6 \times 6 \times 6$ Monkhorst-Pack $k$-point mesh. Raman mode frequencies were computed using the frozen phonon method to capture soft-modes driven by pressure. To find the minimum-energy configuration of the soft modes with pressure, we added one (under $\sim 2 \mathrm{GPa}$ ) or two unstable soft modes (under $\sim 3-5 \mathrm{GPa}$ ) in the ground state structure and moved the atoms according to the conjugate-gradient algorithm until the Hellman-Feynman forces were less than $1.0 \mathrm{meV} / \mathrm{A}$. Interestingly, when we added the two soft modes to the Pnnm ground state under the higher pressures (3-5 GPa), only the $R_{a}$ soft mode leading to the monoclinic $\delta$ phase remains after the relaxation. A Heisenberg-type spin Hamiltonian $\left(\mathcal{H}=-\sum_{\langle i, j\rangle} J_{i, j} S_{i} \cdot S_{j}\right)$ was employed to estimate the exchange interactions. As a point of comparison, the calculated $J$ value at ambient conditions $(-0.12 \mathrm{meV})$ is in good agreement with the experimental value $(-0.07 \mathrm{meV}) .^{34,35}$

Data Availability

Relevant data is available upon request from the corresponding authors, Jan Musfeldt (email: musfeldt@utk.edu) or Jun Hee Lee (email: junhee@unist.ac.kr).

\section{ACKNOWLEDGEMENTS}

This work was supported by the National Science Foundation [DMR-1707846 (JLM, UT) and DMR-1703003 (JLM, EWU)], the Petroleum Research Fund [52052-ND10 (JLM, 
UT)], and the Department of Energy, Office of Science, Basic Energy Sciences, Materials Sciences and Engineering Division (RF). The research at UNIST (JHL, MSK) was supported by Basic Science Research Program through the National Research Foundation of Korea (NRF) funded by the Ministry of Science, ICT \& Future Planning (2015R1C1A1A01055760) and Creative Materials Discovery Program through the National Research Foundation of Korea (NRF) funded by the Ministry of Science and ICT (2017M3D1A1040828). Work at the National Synchrotron Light Source at Brookhaven National Laboratory was funded by the Department of Energy (DEAC98-06CH10886). The use of the U2A beamline was supported by COMPRES under NSF Cooperative Agreement EAR 11-57758 and CDAC (DE-FC03-03N00144). This research used resources of the National Energy Research Scientific Computing Center, a DOE Office of Science User Facility supported by the Office of Science of the U.S. Department of Energy under Contract No. DE-AC02-05CH11231.

\section{AUTHOR CONTRIBUTIONS}

This project was conceived by J.L.M. (UT), J.H.L., R.S.F., Z.L., and K.R.O. The sample was synthesized by J.L.M. (EWU), spectroscopic measurements were performed by K.R.O., Z.L., and J.L.M. (UT), and first-principles calculations and predictions based on densityfunctional theory were carried out by J.H.L., M.K., and R.S.F. All authors discussed the data. The manuscript was written by K.R.O., J.H.L., R.S.F., and J.L.M. (UT), and all authors commented on the document.

\section{ADDITIONAL INFORMATION}

Competing interests: The authors declare no competing financial interests.

Publisher's note: Springer Nature remains neutral with regard to jurisdictional claims in published maps and institutional affiliations.

\section{REFERENCES}

1. Dagotto, E. Complexity in strongly correlated electronic systems. Science 309, 257-262 (2005)

2. Grochala, W., Hoffmann, R., Feng, J. \& Ashcroft, N. W. The chemical imagination at work in very tight places. Angew. Chem. Int. Ed. 46, 3620-3642 (2007).

3. Sato, O., Tao, J. \& Zhang, Y.-Z. Control of magnetic properties through external stimuli. Angew. Chem. Int. Ed. 46, 2152-2187 (2007).

4. Tokura, Y. \& Nagaosa, N. Orbital physics in transition-metal oxides. Science $\mathbf{2 8 8 ,}$ 462-468 (2000)

5. Fortune, N. A. et al. Cascade of magnetic-field-induced quantum phase transitions in a spin $-1 / 2$ triangular-lattice antiferromagnet. Phys. Rev. Lett. 102, 257201 (2009).

6. Kim, J. W. et al. Successive magnetic-field-induced transitions and colossal magnetoelectric effect in $\mathrm{Ni}_{3} \mathrm{TeO}_{6}$. Phys. Rev. Lett. 115, 137201 (2015).

7. Choi, E.-M., Keibeuker, J. E. \& MacManus-Driscoll, J. L. Strain-tuned enhancement of ferromagnetic $T_{C}$ to $176 \mathrm{~K}$ in Sm-doped $\mathrm{BiMnO}_{3}$ thin films and determination of magnetic phase diagram. Sci. Rep. 7, 43799 (2017).

8. Frandsen, B. A. et al. Volume-wise destruction of the antiferromagnetic Mott insulating state through quantum tuning. Nat. Commun. 7, 12519 (2016).

9. Pfleiderer, C., McMullan, G. J., Julian, S. R. \& Lonzarich, G. G. Magnetic quantum phase transition in MnSi under hydrostatic pressure. Phys. Rev. B 55, 8330-8338 (1997).

10. Greiner, M., Mandel, O., Esslinger, T., Hänsch, T. W. \& Bloch, I. Quantum phase transition from a superfluid to a Mott insulator in a gas of ultracold atoms. Nature 413, 39-44 (2002).

11. Caviglia, A. D. et al. Electric field control of the $\mathrm{LaAlO}_{3} / \mathrm{SrTiO}_{3}$ interface ground state. Nature 456, 624-627 (2008).

12. Vinslava, A., Tasiopoulpos, A. J., Wernsdorfer, W., Abboud, K. A. \& Christou, G. Molecules at the quantum-classical nanoparticle interface: giant $M_{70}$ single-molecule magnets of $\sim 4 \mathrm{~nm}$ diameter. Inorg. Chem. 55, 3419-3430 (2016).

13. Furukawa, T., Miyagawa, K., Taniguchi, H., Kato, R. \& Kanoda, K. Quantum criticality of Mott transition in organic materials. Nat. Phys. 11, 221-224 (2015).

14. Büttgen, N. et al. Quantum criticality in transition-metal oxides. J. Low Temp. Phys. 161, 148-166 (2010).

15. Terletska, H., Vučičević, J., Tanasković, D. \& Dobrosavljević, V. Quantum critical transport near the Mott transition. Phys. Rev. Lett. 107, 026401 (2011).

16. Tian, Z. et al. Field-induced quantum metal-insulator transition in the pyrochlore iridate $\mathrm{Nd}_{2} \mathrm{Ir}_{2} \mathrm{O}_{7}$. Nat. Phys. 12, 134-139 (2016).

17. Biscaras, J. et al. Multiple quantum criticality in a two-dimensional superconductor. Nat. Mater. 12, 542-548 (2013).

18. Gegenwart, P. et al. Magnetic-field induced quantum critical point in $\mathrm{YbRh}_{2} \mathrm{Si}_{2}$. Phys. Rev. Lett. 89, 056402 (2002).
19. Joe, Y. I. et al. Emergence of charge density wave domain walls above the superconducting dome in $1 T$-TiSe 2 . Nat. Phys. 10, 421-425 (2014).

20. Landee, C. P. \& Turnbull, M. M. Recent developments in low-dimensional copper (II) molecular magnets. Eur. J. Inorg. Chem. 2013, 2266-2285 (2013).

21. Goddard, P. A. et al. Experimentally determining the exchange parameters of quasi-two-dimensional Heisenberg magnets. New. J. Phys. 10, 083205 (2008).

22. Coronado, E. \& Day, P. Magnetic molecular conductors. Chem. Rev. 104, 5419-5448 (2004).

23. Manson, J. L. et al. Strong H...F bonds and synthons in polymeric quantum magnets: structural, magnetic, and theoretical characterization of $\left[\mathrm{Cu}\left(\mathrm{HF}_{2}\right)(\right.$ pyrazine $\left.)_{2}\right] \mathrm{SbF}_{6}, \quad\left[\mathrm{Cu}_{2} \mathrm{~F}(\mathrm{HF})(\mathrm{HF} 2)(\text { pyrazine })_{4}\right]\left(\mathrm{SbF}_{6}\right)_{2}$, and $\left[\mathrm{CuAg}\left(\mathrm{H}_{3} \mathrm{~F}_{4}\right)(\text { pyrazine })_{5}\right]$ $\left(\mathrm{SbF}_{6}\right)_{2}$. J. Am. Chem. Soc. 131, 6733-6747 (2009).

24. Manson, J. L., Kmety, C. R., Epstein, A. J. \& Miller, J. S. Spontaneous magnetization in the $\mathrm{M}\left[\mathrm{N}\left(\mathrm{CN}_{2}\right)\right]_{2}(\mathrm{M}=\mathrm{Cr}, \mathrm{Mn})$ weak ferromagnets. Inorg. Chem. 38, 2552-2553 (1999).

25. Nuttall, C. J., Takenobu, T., Iwasa, Y. \& Kurmoo, M. Pressure dependence of the magnetization of $\mathrm{M}^{\prime \prime}\left(\mathrm{N}(\mathrm{CN})_{2}\right)_{2}$ : mechanism for the long range magnetic ordering. Mol. Cryst. Liq. Cryst. 343, 227-234 (2000).

26. Jestädt, Th et al. Muon-spin-rotation and magnetization study of metal-organic magnets based on the dicyanamide anion. J. Phys. Condens. Matter. 13, 2263-2270 (2001).

27. Batten, S. R. \& Murray, K. S. Structure and magnetism of coordination polymers containing dicyanimide and tricyanomethanide. Coord. Chem. Rev. 246, 103-130 (2003).

28. Lappas, A., Wills, A. S., Green, M. A., Prassides, K. \& Kurmoo, M. Magnetic ordering in the rutile molecular magnets $M^{\prime \prime}\left[\mathrm{N}(\mathrm{CN})_{2}\right]_{2}\left(M=\mathrm{Ni}, \mathrm{Co}, \mathrm{Fe}, \mathrm{Mn}, \mathrm{Ni}_{0.5} \mathrm{Co}_{0.5}\right.$, and $\mathrm{Ni}_{0.5} \mathrm{Fe}_{0.5}$ ). Phys. Rev. B 67, 144406 (2003).

29. Demchenko, D. O. et al. Electronic structure of the transition-metal dicyanimides $M\left[N(C N)_{2}\right]_{2}(M=M n, F e, C o, N i, C u)$. Phys. Rev. B 69, 205105 (2004).

30. Brinzari, T. V. et al. Electron-phonon and magnetoelastic interactions in ferromagnetic $\mathrm{Co}\left[\mathrm{N}\left(\mathrm{CN}_{2}\right)\right]_{2}$. Phys. Rev. Lett. 111, 047202 (2013).

31. Musfeldt, J. L. et al. Pressure-induced local lattice distortions in $a-\operatorname{Co}\left[\mathrm{N}(\mathrm{CN})_{2}\right]_{2}$. Inorg. Chem. 52, 14148-14154 (2013).

32. Yakovenko, A. A., Chapman, K. W. \& Halder, G. J. Pressure-induced structural phase transformation in cobalt(II) dicyanamide. Acta Cryst. B71, 252-257 (2015).

33. Musfeldt, J. L. et al. Pressure-temperature phase diagram reveals spin-lattice interactions in $\mathrm{Co}\left[\mathrm{N}\left(\mathrm{CN}_{2}\right)\right]_{2}$. Inorg. Chem. 56, 4950-4955 (2017).

34. Kmety, C. R. et al. Noncollinear antiferromagnetic structure of the molecule-based magnet $\mathrm{Mn}[\mathrm{N}(\mathrm{CN} 2)]_{2}$. Phys. Rev. B 62, 5576-5588 (2000).

35. Manson, J. L., Kmety, C. R., Palacio, F., Epstein, A. J. \& Miller, J. S. Low-field remanent magnetization in the weak ferromagnet $\mathrm{Mn}\left[\mathrm{N}(\mathrm{CN})_{2}\right]_{2}$. Evidence for spin-flop behavior. Chem. Mater. 13, 1067-1073 (2001).

36. Brinzari, T. V. et al. Quantum critical transition amplifies magnetoelastic coupling in $\mathrm{Mn}\left[\mathrm{N}(\mathrm{CN})_{2}\right]_{2}$. Phys. Rev. Lett. 110, 237202 (2013).

37. Quintero, P. A. et al. Pressure-induced enhancement of the magnetic anisotropy in $\mathrm{Mn}\left[\mathrm{N}\left(\mathrm{CN}_{2}\right)\right]_{2}$. Phys. Rev. B 91, 014439 (2015).

38. Brinzari, T. V. et al. Local lattice distortions in $\mathrm{Mn}\left[\mathrm{N}\left(\mathrm{CN}_{2}\right)\right]_{2}$ under pressure. Inorg. Chem. 55, 1956-1961 (2016).

39. Rondinelli, J. M. \& Fennie, C. J. Octahedral rotation-induced ferroelectricity in cation ordered perovskites. Adv. Mater. 24, 1961-1968 (2012).

40. Ye, F. et al. Magnetic order and spin-flop transitions in the cobalt-doped multiferroic $\mathrm{Mn}_{1-x} \mathrm{Co}_{x} \mathrm{WO}_{4}$. Phys. Rev. B 86, 094429 (2012).

41. Manson, J. L. et al. Magnetic structure and exchange interactions in quasi-onedimensional $\mathrm{MnCl}_{2}$ (urea) ${ }_{2}$. Inorg. Chem. 54, 11898-11905 (2015).

42. McConnell, A. C., Bell, J. D. \& Miller, J. S. Pressure-induced transition from an antiferromagnet to a ferrimagnet for $\mathrm{Mn}^{\prime \prime}(\mathrm{TCNE})\left[\mathrm{C}_{4}(\mathrm{CN})_{8}\right]_{1 / 2}$ (TCNE = tetracyanoethylene). Inorg. Chem. 51, 9978-9982 (2012).

43. Duboc, C. Determination and prediction of the magnetic anisotropy of $\mathrm{Mn}$ ions. Chem. Soc. Rev. 45, 5834-5847 (2016).

44. Jain, P. et al. Multiferroic behavior associated with an order-disorder hydrogen bonding transition in metal-organic frameworks (MOFs) with the perovskite $\mathrm{ABX}_{3}$ architecture. J. Am. Chem. Soc. 131, 13625-13627 (2009).

45. Jain, P. et al. Switchable electric polarization and ferroelectric domains in a metalorganic-framework. npj Quant. Mater. 1, 16012 (2016).

46. Xin, L. et al. Growth of centimeter-sized $\left[\left(\mathrm{CH}_{3}\right)_{2} \mathrm{NH}_{2}\right]\left[\mathrm{Mn}(\mathrm{HCOO})_{3}\right]$ hybrid formate perovskite single crystals and Raman evidence of pressure-induced transitions. New. J. Chem. 41, 151-159 (2017).

47. Clune, A. J. et al. Magnetic field-temperature phase diagram of multiferroic $\left[\left(\mathrm{CH}_{3}\right)_{2} \mathrm{NH}_{2}\right] \mathrm{Mn}(\mathrm{HCOO})_{3}$. Phys. Rev B. 96, 104424 (2017).

48. Silverstein, H. J. et al. Incommensurate crystal supercell and polarization flop observed in the magnetoelectric ilmenite $\mathrm{MnTiO}_{3}$. Phys. Rev. B 93, 054416 (2016).

49. Ross, N. L., Ko, J. \& Prewitt, C. T. A new phase transition in $\mathrm{MnTiO}_{3}: \mathrm{LuNbO}_{3}{ }^{-}$ perovskite structure. Phys. Chem. Miner. 16, 621-629 (1989). 
50. Longo, J. M. \& Kafalas, J. A. Effect of pressure on the crystal structure of $\mathrm{CsMnCl}_{3}$ and $\mathrm{RbMnCl}_{3}$. J. Sol. State Chem. 3, 429-433 (1971).

51. Kedzie, R. W., Shane, J. R., Kestigan, M. \& Croft, W. J. Resonance observation of antiferromagnetic ordering in $\mathrm{RbMnCl}_{3}, \mathrm{CsMnCl}_{3}$, and $\mathrm{KMnCl}_{3}$. J. Appl. Phys. 36, 1195-1196 (1965).

52. Mao, H. K., Bell, P. M., Shaner, J. W. \& Steinberg, D. J. Specific volume measurements of $\mathrm{Cu}, \mathrm{Mo}, \mathrm{Pd}$, and $\mathrm{Ag}$ and calibration of the ruby $R_{1}$ fluorescence pressure gauge from 0.06 to 1 Mbar. J. Appl. Phys. 49, 3276-3283 (1978).

53. Loschen, C., Carrasco, J., Neyman, K. M. \& Illas, F. First-principles LDA+U and GGA $+U$ study of cerium oxides: dependence on the effective $U$ parameter. Phys. Rev. $B$ 75, 035115 (2007).

54. Perdew, J. P., Burke, K. \& Ernzerhof, M. Generalized gradient approximation made simple. Phys. Rev. Lett. 77, 3865-3868 (1996).

55. Kresse, G. \& Hafner, J. Ab initio molecular dynamics for liquid metals. Phys. Rev. $B$ 47, R558-R561 (1993).

56. Kresse, G. \& Furthmüller, J. Efficient iterative schemes for $a b$ initio total-energy calculations using a plane-wave basis set. Phys. Rev. B 54, 11169-11186 (1996).

57. Dudarev, S. L., Botton, G. A., Savrasov, S. Y., Humphreys, C. J. \& Sutton, A. P. Electron-energy-loss spectra and the structural stability of nickel oxide: an LSDA +U study. Phys. Rev. B 57, 1505-1509 (1998).
58. Blöchl, P. E. Projector augmented-wave method. Phys. Rev. B 50, 17953-17979 (1994).

59. Kresse, G. \& Joubert, D. From ultrasoft pseudopotentials to the projector augmented-wave method. Phys. Rev. B 59, 1758-1775 (1999).

Open Access This article is licensed under a Creative Commons Attribution 4.0 International License, which permits use, sharing, adaptation, distribution and reproduction in any medium or format, as long as you give appropriate credit to the original author(s) and the source, provide a link to the Creative Commons license, and indicate if changes were made. The images or other third party material in this article are included in the article's Creative Commons license, unless indicated otherwise in a credit line to the material. If material is not included in the article's Creative Commons license and your intended use is not permitted by statutory regulation or exceeds the permitted use, you will need to obtain permission directly from the copyright holder. To view a copy of this license, visit http://creativecommons. org/licenses/by/4.0/.

(c) The Author(s) 2017 